\title{
DETECÇÃO DE VAGAS E ESTACIONAMENTO AUTÔNOMO DE VEÍCULOS
}

\author{
Marcos G. Prado*, André Chaves Magalhães ${ }^{\dagger}$, Denis F. Wolf*, Valdir Grassi JR ${ }^{\dagger}$ \\ * Laboratório de Robótica Móvel \\ Instituto de Ciências Matemáticas e de Computação - Universidade de São Paulo \\ São Carlos, SP, Brasil \\ ${ }^{\dagger}$ Laboratório de Sistemas Inteligentes \\ Escola de Engenharia de São Carlos - Universidade de São Paulo \\ São Carlos, SP, Brasil
}

Emails: mgprado@icmc.usp.br, acmagalhaes@usp.br, denis@icmc.usp.br, vgrassi@usp.br

\begin{abstract}
Autonomous navigation is one of the fundamental problems in mobile robotics. This problem has been addressed for decades and still has great potential for scientific research. Most solutions and algorithms developed in this field is designed for robots that operate in structured environments. However, another issue of great interest to researchers in this area is autonomous navigation in outdoor environments. In partially structured environments autonomous vehicles (large robots) must be able to avoid obstacles that may arise along the way. This paper addresses the development of an intelligent system able to generate and run a path planning for parking of autonomous vehicles in semi-structured environments. The system is able to recognize parking lots using sensors installed in the vehicle, generate a valid path that leads up to the parking lot and send the steering commands and acceleration that to guide the vehicle to its goal point.
\end{abstract}

Keywords - path planning, autonomous vehicles, parking of autonomous vehicles, and search-based planning.

\begin{abstract}
Resumo- Navegação autônoma é um dos problemas fundamentais na área de robótica móvel. Esse problema vem sendo pesquisado nessa área por décadas e ainda apresenta um grande potencial para pesquisas científicas. A maior parte dos algoritmos e soluções desenvolvidas nessa área foi concebida para que robôs operem em ambientes estruturados. No entanto, outra questão de grande interesse para pesquisadores da área é a navegação em ambientes externos. Em ambientes não estruturado os veículos autônomos (robôs de grande porte) devem ser capazes de desviar de obstáculos, que eventualmente apareçam no caminho. Este artigo aborda o desenvolvimento de um sistema inteligente capaz de gerar e executar um planejamento de caminho para o estacionamento de veículos autônomos em ambientes semi-estruturados. O sistema é capaz de reconhecer vagas de estacionamento por meio de sensores instalados no veículo, gerar uma trajetória válida que o conduza até a vaga e enviar os comandos de esterçamento e aceleração que guiam o veículo pelo caminho gerado.
\end{abstract}

Palavras-chave- planejamento de trajetória, veículos autônomos, estacionamento de veículo autônomo, e planejamento baseado em busca.

\section{Introdução}

A pesquisa em robótica móvel tem alcançado um progresso significativo nos últimos 20 anos. Parte dela se especializa em navegação autônoma, que é uma tarefa fundamental em robôs móveis. Nesses anos, diversas abordagens têm sido direcionadas para a navegação autônoma em ambientes externos, onde uma das maiores preocupações é determinar as regiões navegáveis para que possa ser desempenhada uma navegação autônoma com segurança. Nesse contexto, a tarefa dos robôs móveis tem sido estendida para veículos robóticos inteligentes e uma aplicação dessa tecnologia é o desenvolvimento de sistemas de direção autônoma.

Os trabalhos relacionados aos veículos autônomos inteligentes têm crescido tanto no setor acadêmico como na indústria. Mapeamento de ambientes, localização, planejamento e controle são problemas complexos e totalmente ligados à pesquisa de veículos autônomos inteligentes. Trabalhos como (Markelic et al., 2011), (Petrovskaya and Thrun, 2009), (Bonnifait et al., 2008) e (Guizzo, 2011), têm focado na nave- gação de veículos robóticos em ambientes urbanos. Competições tais como DARPA Grand Challenge (Montemerlo et al., 2006) e Urban Challenge (Buehler et al., 2009), e ELROB (Schneider and Wildermuth, 2011) têm impulsionado o estado da arte no controle de veículos autônomos.

O aumento do número de veículos, a imprudência dos condutores e o alto índice de acidentes são exemplos de problemas no trânsito atualmente. Em um intervalo de dez anos, o crescimento foi de aproximadamente $125 \%$ na frota do país segundo DENATRAN ${ }^{1}$. Dentre alguns pontos negativos referentes a este crescimento pode ser citada a dificuldade de encontrar vagas livres para estacionamento, tanto públicas como privadas. Além disso, por muitas vezes existe a dificuldade de realização da manobra de estacionamento em determinadas vagas, principalmente por idosos e motoristas principiantes. Esse artigo apresenta um sistema de estacionamento para veículos autônomos, capaz de reconhecer vagas, planejar e executar trajetórias.

Diferentes abordagens para detectar a vaga de

\footnotetext{
${ }^{1}$ http://www.denatran.gov.br/frota.htm
} 
estacionamento tem sido propostas, bem como diferentes tipos de sensores são usados para essa detecção. Os simuladores apresentados em (Osório et al., 2001) e (Andrade, 2011) fazem uso de sensores do tipo laser para reconhecimento de vagas, e ambos utilizam Redes Neurais Artificiais (RNA) na construção do controlodor de trajetória. Em (Han et al., 2011) é proposto um algoritmo baseado em árvores aleatórias de exploração rápida (LaValle and Kuffner, 2001) para a criação de trajetórias específicas para estacionamento. Em ambientes reais, o trabalho de (Honório et al., 2010) aborda o problema de estacionamento em vagas paralelas com metodologia baseada em lógica fuzzy, enquanto que (Jeevan et al., 2010) e (Likhachev and Ferguson, 2009) tratam do planejamento de trajetória para vagas perpendiculares.

Diferente dos trabalhos citados anteriormente, a abordagem apresentada nesse artigo permite o estacionamento em diferentes tipos de vagas e foi validada em situações reais.

O trabalho apresentado aqui faz parte de um projeto maior que tem como objetivo a criação de veículos autônomos inteligentes para navegação em ambientes urbanos. O projeto CaRINA (Carro Robótico Inteligente para Navegação Autônoma) é desenvolvido pelo Laboratório de Robótica Móvel (LRM-ICMC/USP) em colaboração com o Laboratório de Sistemas Inteligentes (LASIEESC/USP) e INCT-SEC (Instituto Nacional de Ciência e Tecnologia em Sistemas Embarcados Críticos).

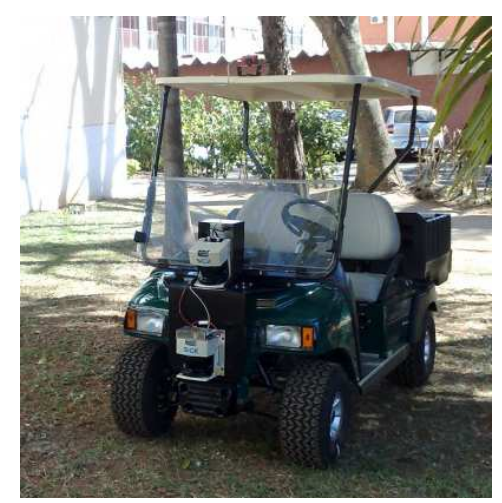

Figura 1: Veículo Elétrico CaRINA I.

\section{Veículo Autônomo}

O veículo elétrico automatizado denominado CaRINA I (Figura 1), utilizado neste trabalho, é equipado de um sensor de medida laser SICK LMS 291 , com alcance de 80 metros e ângulo de varredura de $180^{\circ}$, instalado na parte frontal, e de dois sensores Hokuyo UTM-30LX, cada um com alcance de 30 metros e ângulo de varredura de $270^{\circ}$, instalados nas duas laterais na parte traseira. As informações provenientes desses três sensores foram combinadas de forma a se obter uma única nuvem de pontos em um ambiente de duas dimensões. Dessa forma, para o sistema de navegação é como se existisse apenas um sensor laser com alcance de 30 metros capaz de medir distâncias a cada grau nos $360^{\circ}$ ao redor do veículo. Esse laser virtual é utilizado neste trabalho para a detecção de obstáculos, identificação das vagas de estacionamento, localização e mapeamento. A Figura 2 ilustra o sensor laser virtual gerado pela fusão dos três sensores reais.

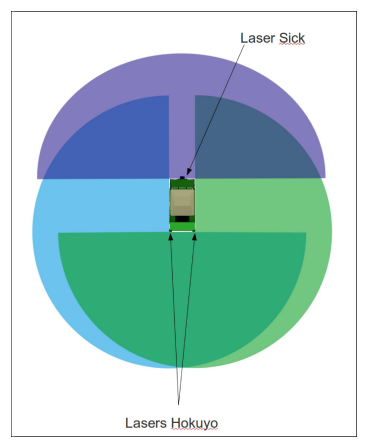

(a)

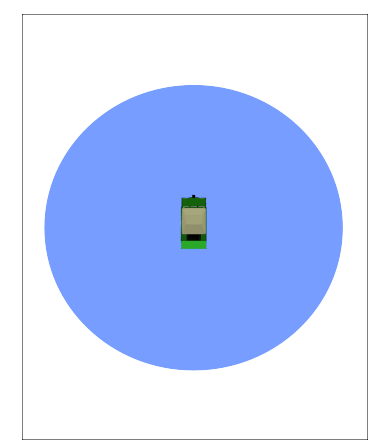

(b)
Figura 2: Visão superior do veículo mostrando em (a) o campo de visão dos três sensores laser, e em (b) a representação do laser virtual.

A localização do veículo é estimada a partir da união de dois sistemas de localização. O primeiro estima a posição utilizando a informação de dois encoders e uma IMU (do inglês Inertial Measurement Unit). Um dos encordes foi acoplado a umas das rodas dianteiras para medir seu giro, e outro acoplado no barramento de direção para estimar o esterçamento das rodas. A IMU, posicionada no centro do eixo traseiro, permite estimar a orientação do veículo. Este primeiro sistema calcula a odometria baseando-se no modelo Ackerman. O segundo sistema utiliza o algoritmo ICP (do inglês Iterative Closest/Corresponding Point) (Censi, 2008) para fazer um casamento dos dados dos sensores laser durante o deslocamento do veículo para estimar sua posição atual. Um filtro de Kalman estendido (Welch and Bishop, 1995) é utilizado para combinar as estimativas dos dois sistemas de localização, reduzindo imprecisão e filtrando erros de medida.

\section{Planejamento e Controle}

O planejador é responsável por definir uma trajetória entre a configuração atual do veículo e a configuração de destino, evitando colisões. O algoritmo utilizado neste trabalho é baseado em malhas de estado, e descrito em maiores detalhes em (Likhachev and Ferguson, 2009), e sua implementação para o CaRINA I, descrita em (Magalhães 
et al., 2013). Esse algoritmo foi escolhido devido (I) a sua capacidade de atuar em um ambiente parcialmente conhecido; (II) seu modelo de espaço de busca que discretiza o ambiente de acordo as limitações cinemática do veículo; (III) e sua capacidade de encontrar trajetórias válidas dentro de um tempo predefinido. Essas características destacadas tornam-no viável para atuar em ambientes reais, visto que as mesmas estão presentes nesse ambiente.

Essa abordagem trabalha sobre um ambiente discretizado construído por meio de um conjunto de movimentos primitivos válidos, que combinados formam uma malha de estados. Neste trabalho, o estado do veículo é definido por sua posição e orientação, $(x, y, \theta)$, e a malha de estados foi contruída a partir de quatorze movimentos primitivos, sendo sete movimentos para a frente e sete para trás (Figura 3) combinados entre si em 24 ângulos de orientação diferentes. O conjunto de movimentos primitivos foi criado discretizando valores de velocidade de translação e esterçamento, e aplicando essas entradas no modelo cinemático Ackerman para os parâmetros do veículo CaRINA I.

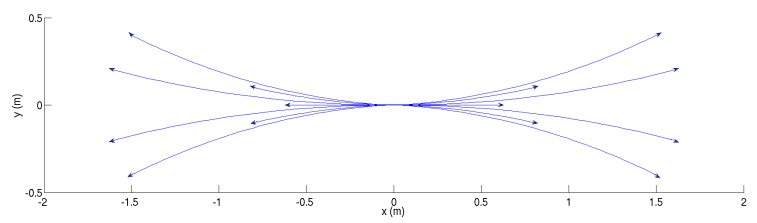

Figura 3: Conjunto de movimentos primitivos. 14 ações de movimentos válidos para o veículo ( 7 movimentos para frente e 7 para traz).

Utilizando a malha de estados criada, o planejador busca uma trajetória até o destino através de um algoritmo $\mathrm{AD}^{*}$. Em seguida o sistema de controle envia para o veículo os comando de esterçamento e aceleração para seguir a trajetória. Durante a execução da trajetória, o veículo pode detectar obstáculos não mapeados, e para desviar localmente destes obstáculos foi utilizada uma abordagem baseada no método de janela dinâmica (DWA - Dynamic Window Approach) (Fox et al., 1997). Essa abordagem gera trajetórias locais para o veículo através de simulações do seu próximo estado para um conjunto de amostras de entradas de controle. Dentre as várias trajetórias simuladas, aquela que apresentar o menor custo segundo um critério adotado será enviada ao veículo, sendo que as simulações que colidem com obstáculos são eliminadas. Esse custo é calculado utilizando-se uma ponderação da proximidade da trajetória aos obstáculos, proximidade com a meta, e proximidade com a trajetória global inicialmente obtida pelo planejador.

\section{Detecção de Vagas de Estacionamento}

O sistema de estacionamento autônomo é capaz de detectar um espaço e classificá-lo como uma possível vaga de estacionamento. Para isso, as dimensões das vagas são predefinidas e o veículo utiliza o conjunto de sensores laser (conforme descrito anteiormente) para estimar a posição desses espaços baseado nos obstáculos presentes no ambiente. Inicialmente o problema foi definido com as seguintes especificações: (i) ambiente de duas dimensões; (ii) ambiente específico de estacionamento, onde os obstáculos são veículos, paredes ou colunas; (iii) três tipos de vaga - perpendicular, paralela/baliza, diagonal (45 graus); (iv) possíveis vagas apenas dos lados direito ou esquerdo do veículo; (v) obstáculos alinhados com a zona navegável.

O sistema de detecção inicialmente agrupa os feixes de laser vizinhos que possuem uma diferença de tamanho pequena predefinida, considerando esses grupos como possíveis referências para a busca das vagas. Após esse agrupamento é feita uma filtragem para eliminar obstáculos distantes e também obstáculos pequenos (poucos feixes agrupados). Em seguida são feitas varreduras com o laser nas proximidades dos obstáculos classificados como válidos, buscando um espaço livre, correspondente a uma vaga de estacionamento. Esse espaço é definido de acordo com o tipo de vaga buscado, sendo que o sistema tem suporte a três tipos, como citado anteriormente. Ao encontrar um espaço livre, uma posição de destino é definida e o sistema de planejamento é responsável por gerar uma trajetória até a mesma.

A Figura 4 ilustra a detecção das vagas de estacionamento. Os pontos vermelhos são os obstáculos identificados por meio do laser. A vaga é encontrada e um ponto de destino, representado por uma seta na imagem, é gerado baseado no tipo de vaga buscada. Os obstáculos detectados pelo laser nas figuras 4(a) e 4(b), são veículos reais, enquanto na figura 4(c) a coleta foi realizada em um ambiente simulado.

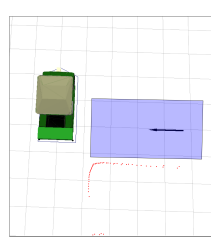

(a)

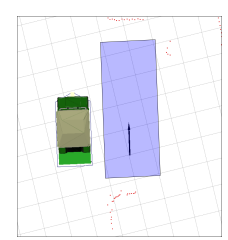

(b)

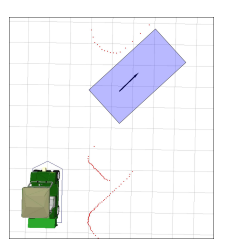

(c)
Figura 4: Detecção de vagas de estacionamento. Os retângulos em azul representam os espaços definidos como vagas de estacionamento. (a) Vaga perpendicular. (b) Vaga paralela. (c) Vaga diagonal. 


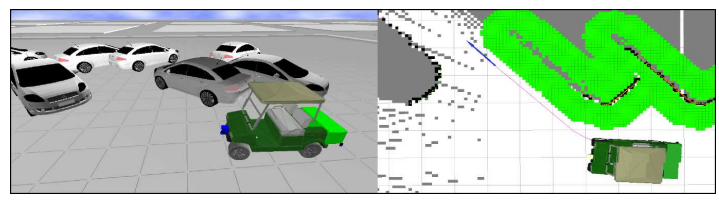

(a)

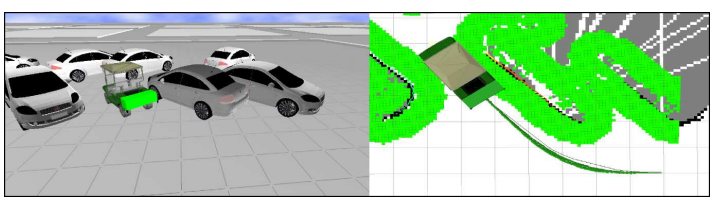

(b)

Figura 5: Sistema de estacionamento autônomo em ambiente simulado - Estacionamento diagonal. (a) Mapeamento, detecção da vaga de estacionamento e planejamento de trajetória. (b) Execução e finalização da manobra.

\section{$5 \quad$ Resultados}

O sistema de detecção de vagas e de estacionamento autônomo foi implementado utilizando a plataforma ROS (Robot Operating System). Foram feitos testes em ambiente simulado utilizando um modelo do CaRINA I implementado para o simulador Gazebo. Nas simulações, os obstáculos para referência às vagas de estacionamento eram constituídos por outros veículos.

As Figuras 5, 6, e 7 mostram alguns dos resutados obtidos através de simulação para estacionamento diagonal, perpendicular e paralelo, respectivamente. O lado esquerdo de cada figura ilustra o ambiente simulado, enquanto o lado oposto, a visualização do ambiente na perspectiva dos sensores. A área branca denota zona navegável, a parte preta indica presença de obstáculos, a área cinza é configurada como desconhecida e a verde é uma área de segurança por estar próximo de obstáculos, e que tem seu custo local elevado pelo método DWA. Nos experimentos, após a detecção da vaga de estacionamento, a posição e orientação final desejada para o veículo é definida pelo sistema e marcada nas figuras pela seta azul. As trajetórias planejada e executada também são indicadas nas figuras. É interessante ressaltar que no ambiente simulado o sistema de localização foi composto apenas pela nuvem de pontos criada por meio dos sensores lasers. Nos testes de estacionamento em vagas paralelas o sistema conseguiu uma melhor performance quando o obstáculo de referência (outro veículo estacionado) estava posicionado entre 0.5 e 1.5 metros da lateral do veículo.

\subsection{Análise dos Resultados Simulados}

Os resultados simulados foram analisados, e utilizou-se a porcentagem de manobras válidas para validação do sistema. Para a classificação de uma manobra como válida, levou-se em consi-

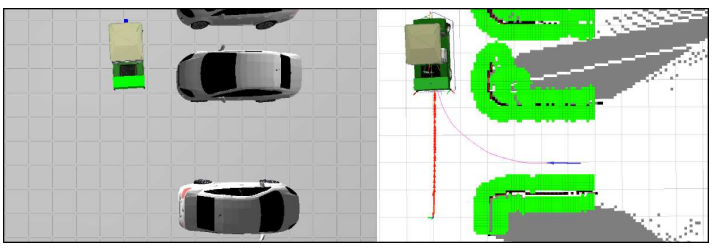

(a)



(b)

Figura 6: Sistema de estacionamento autônomo em ambiente simulado - Estacionamento perpendicular. (a) Mapeamento, detecção da vaga de estacionamento e planejamento de trajetória. (b) Execução e finalização da manobra.

deração as dimensões da vaga de estacionamento e as dimensões do veículo. Dessa forma, uma manobra é classificada como inválida quando após sua finalização parte do veículo permanece fora dos limites da vaga.

Foi analisado um total de 30 manobras de estacionamento simuladas, sendo 10 manobras para cada tipo de vaga. Desse total, $83 \%$ foram classificadas como válidas, ou seja, foram finalizadas com sucesso. As manobras em vagas paralelas e perpendiculares obtiveram $80 \%$ de sucesso, enquanto que as manobras em vagas diagonais alcançaram $90 \%$ de acerto (Tabela 1 ).

Tabela 1: Validação das manobras de estacionamento

\begin{tabular}{|c|l|l|c|}
\hline Tipo de vaga & $\begin{array}{l}\text { Manobras } \\
\text { válidas }\end{array}$ & $\begin{array}{l}\text { Manobras } \\
\text { inválidas }\end{array}$ & Total \\
\hline Perpendicular & 8 & 2 & 10 \\
\hline Paralela & 8 & 2 & 10 \\
\hline Diagonal & 9 & 1 & 10 \\
\hline
\end{tabular}

Em todas as 30 manobras analisadas o sistema de planejamento foi capaz de construir uma trajetória consistente. As manobras classificadas como inválidas podem ter sido afetadas pelo erro do sistema de localização em conjunto com as limitações do sistema de controle baseado em DWA. Visto que o controle faz uso de um conjunto discretizado de velocidades, causando em alguns momentos uma leve fuga do caminho global. Tanto a manobra em vagas paralelas como em vagas perpendiculares podem ser consideradas mais complexas do que a manobra em vaga diagonal. A última pode ser realizada a partir de uma curva mais suave, devido sua orientação em relação ao posicionamento inicial do veículo. Dessa forma, o sistema de localização permanece mais consistente. 


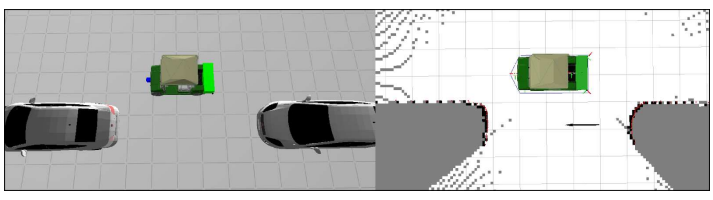

(a)

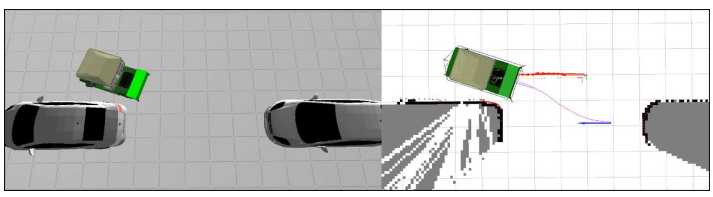

(b)

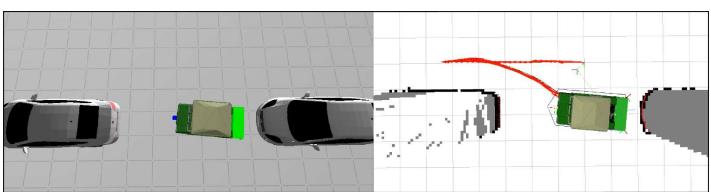

(c)

Figura 7: Sistema de estacionamento autônomo em ambiente simulado - Estacionamento paralelo. (a) Mapeamento e detecção da vaga de estacionamento. (b) Planejamento de trajetória. (c) Execução e finalização da manobra.

Enquanto que a manobra em vaga perpendicular necessita de, no mínimo, uma curva de $90^{\circ}$. E a manobra em vaga paralela é composta por duas curvas.

O espaço de atuação do veículo foi discretizado. O mapa utilizado possui resolução de 0.1 metro e o conjunto de movimentos primitivos trabalha com a discretização da orientação em 0.26 radianos. O sistema conseguiu garantir, nas manobras válidas, que o ponto de referência do veículo sempre permanecesse dentro de um limite máximo de $0.2 \mathrm{~m}$ de distância do ponto de destino e com uma diferença menor do que 0.26 radianos entre orientação do veículo e a orientação desejada.

\subsection{Resultados preliminares em ambiente real}

O sistema de detecção de vagas e estacionamento também foi testado em ambiente real para vagas perpendiculares e paralelas. Nos testes, a aceleração ficou sob a responsabilidade do condutor pois o veículo ainda não possui o controle de velocidade, apenas o controle de esterçamento. Durante a realização do teste o veículo foi capaz de percorrer o trajeto proposto e estacionar de forma adequada nas vagas.

A Figura 8 apresenta um teste do sistema de planejamento realizado em ambiente real tratando o problema de estacionamento em vaga perpendicular. A Figura 9 apresenta um teste tratando o problema de estacionamento em vaga paralela, a qual está delimitada por duas caixas devidamente posicionadas. Para ambos os casos o veículo concluiu com sucesso o estacionamento.

\section{Conclusão}

Esse trabalho contribui na resolução do problema de planejamento de trajetória para estacionamento de veículos autônomos, atuando em três tipos de vaga: paralela, perpendicular $\left(90^{\circ}\right)$ e diagonal $\left(45^{\circ}\right)$. Várias abordagens tratam da tarefa de estacionamento autônomo, tanto no campo acadêmico como na indústria, mas ainda não validaram uma abordagem que consiga atuar de forma unificada nos três modelos de vaga em ambientes reais. Os produtos comerciais geralmente atuam em situações bastante específicas, limitando tipos de vaga e necessitando de intervenção humana em alguns momentos, por exemplo.

Foram realizados diversos testes experimentais em ambientes reais com a plataforma robótica CaRINA 1 para validar a abordagem descrita.

Como trabalho futuro pretende-se desenvolver um sistema de mapeamento e navegação mais amplo que permita que o veículo procure por vagas em ambientes amplos e complexos.

\section{Agradecimentos}

Os autores agradecem o apoio financeiro da FAPESP, Capes e CNPq.

\section{Referências}

Andrade, K. O. (2011). Sistema neural reativo para o estacionamento paralelo com uma única manobra em veículos de passeio, Dissertação de mestrado, Escola de Engenharia de São Carlos - Universidade de São Paulo, São Carlos-SP.

Bonnifait, P., Jabbour, M. and Cherfaoui, V. (2008). Autonomous navigation in urban areas using gis-managed information, International Journal of Vehicle Autonomous Systems 6(1-2): 83-103. DOI: 10.1504/IJVAS.2008.016479

Buehler, M., Iagnemma, K. and Singh, S. (eds) (2009). The DARPA Urban Challenge: Autonomous Vehicles in City Traffic, George Air Force Base, Victorville, California, USA, Vol. 56 of Springer Tracts in Advanced Robo-tics, Springer. DOI: 10.1007/978-3-642-03991-1

Censi, A. (2008). An ICP variant using a pointto-line metric, Proceedings of the IEEE International Conference on Robotics and Automation (ICRA), Pasadena, CA.

Fox, D., Burgard, W. and Thrun, S. (1997). The dynamic window approach to collision avoidance, Robotics Automation Magazine, IEEE 4(1): 23 -33. DOI: 10.1109/100.580977

Guizzo, E. (2011). How google's self-driving car works. IEEE Spectrum. 


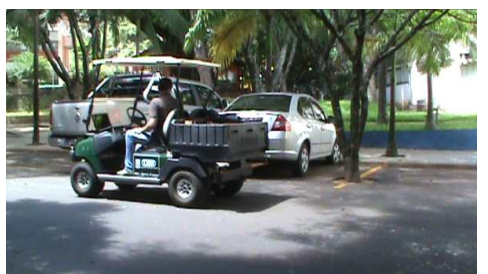

(a)



(b)

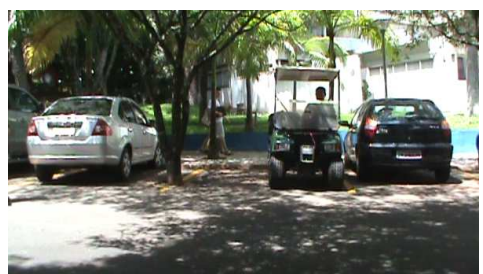

(c)

Figura 8: Teste de estacionamento em vaga perpendicular em ambiente ambiente real. (a) Inicio da manobra de estacionamento. (b) Execução da tragetória encotrada. (c) Finalização da trajetória.



(a)

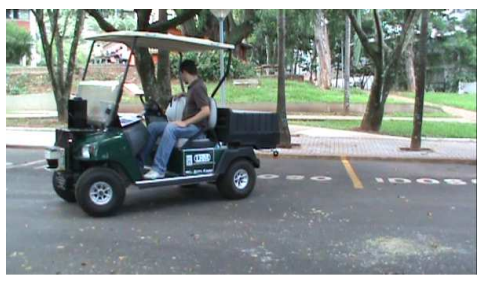

(b)

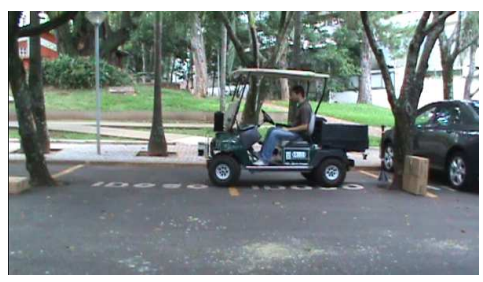

(c)

Figura 9: Teste de estacionamento em vaga paralela em ambiente ambiente real. (a) Inicio da manobra de estacionamento. (b) Execução da tragetória encotrada. (c) Finalização da trajetória.

Han, L., Do, Q. H. and Mita, S. (2011). Unified path planner for parking an autonomous vehicle based on rrt, Robotics and Automation (ICRA), 2011 IEEE International Conference on Robotics and Automation, pp. 5622 -5627. DOI: 10.1109/ICRA.2011.5980105

Honório, L. M., Luiz L. G. Vermma and, L. M. G. and Vidigal, M. (2010). Uma metodologia para aprendizado supervisionado aplicada em veículos inteligentes, XVIII Congresso Brasileiro de Automática .

Jeevan, P., Harchut, F., Mueller-Bessler, B. and Huhnke, B. (2010). Realizing autonomous valet parking with automotive grade sensors, Intelligent Robots and Systems (IROS), 2010 IEEE/RSJ International Conference on, pp. $3824-3829$.

LaValle, S. M. and Kuffner, J. J. (2001). Randomized kinodynamic planning, The International Journal of Robotics Research 20(5): 378-400. OI: 10.1177/02783640122067453

Likhachev, M. and Ferguson, D. (2009). Planning long dynamically feasible maneuvers for autonomous vehicles, The International Jour-nal of Robotics Research 28: 933-945. DOI: $10.1177 / 0278364909340445$

Magalhães, A. C., Prado, M., Grassi Jr, V. and Wolf, D. F. (2013). Autonomous vehicle navigation in semi-structured urban environment, Proceedings of the 2013 IFAC Intelligent Autonomous Vehicle Symposium.

Markelic, I., Kjaer-Nielsen, A., Pauwels, K., Jensen, L. B. W., Chumerin, N., Vidugiriene, A.,
Tamosiunaite, M., Hulle, M. V., Kruerger, N., Rotter, A. and Woergoetter, F. (2011). The driving school system: Learning automated basic driving skills from a teacher in a real car, International Journal of Vehicle Autonomous Systems .

Montemerlo, M., Thrun, S., Dahlkamp, H. and Stavens, D. (2006). Winning the darpa grand challenge with an ai robot, In Proceedings of the AAAI National Conference on Artificial Intelligence, pp. 17-20.

Osório, F. S., Heinen, F. J. and Fortes, L. (2001). Controle inteligente de veículos autônomos: Automatização do processo de estacionamento de carros, X Seminário de Computação (SEMINCO) - FURB.

Petrovskaya, A. and Thrun, S. (2009). Model based vehicle detection and tracking for autonomous urban driving, Autonomous Robots Journal 26(2-3): 123-139. DOI: 10.1007/s10514-009-9115-1

Schneider, F. and Wildermuth, D. (2011). Results of the european land robot trial and their usability for benchmarking outdoor robot systems, in R. Groß, L. Alboul, C. Melhuish, M. Witkowski, T. Prescott and J. Penders (eds), Towards Autonomous Robotic Systems, Vol. 6856 of Lecture Notes in Computer Science, Springer Berlin Heidelberg, pp. 408409.

Welch, G. and Bishop, G. (1995). An introduction to the kalman filter. 